\title{
Communication A Combination of Transmission Line Models as Design Instruments for Electromagnetically Coupled Microstrip Patch Antennas in the $2.45 \mathrm{GHz}$ ISM Band
}

\author{
Ruben Del-Rio-Ruiz (D), Student Member, IEEE, Juan-Manuel Lopez-Garde (D), Jon Legarda (D), \\ Olivier Caytan (D), Member, IEEE, and Hendrik Rogier (D), Senior Member, IEEE
}

\begin{abstract}
This communication presents an analytical framework that combines transmission line models for the design of electromagnetically coupled microstrip patch antennas for the 2.45 GHz industrial, scientific and medical band. It provides initial values for all dimensions of the antenna, with measured resonance frequency errors below $6 \%$. The initial design is optimized in two subsequent phases to center the resonance frequency and to increase the impedance bandwidth $(\mathrm{BW})$, obtaining measured resonance frequency errors below $0.6 \%$ and BW enhancements of more than 1.2 times the original ones, respectively. The model has been validated with antenna prototypes based on rigid and textile materials, exhibiting excellent freespace measured BW of $4 \%$ and $5.12 \%$, maximal measured gains of $4.28 \mathrm{dBi}$ and $7.33 \mathrm{dBi}$, and radiation efficiencies of $63.4 \%$ and $\mathbf{7 1 . 8 \%}$, respectively. Moreover, very stable on-body performance is obtained, with minimal frequency detuning when deploying the textile antenna on the human body. The measured maximum onbody gain for the textile antenna equals $5.5 \mathrm{dBi}$, with a simulated specific absorption rate of $0.323 \mathrm{~W} / \mathrm{kg}$ at $2.45 \mathrm{GHz}$.
\end{abstract}

Index Terms-Design methodology, electromagnetic coupling, flexible structures, microstrip textile antennas, transmission line models.

\section{INTRODUCTION}

$\mathbf{M}$ ICROSTRIP patch antennas (MPAs) have been extensively developed for military [1] and civil [2] applications. Their main characteristics of light weight, planar profile, robustness, ease of fabrication and conformability make them suitable for textile material implementations in body-centric wireless communication (BCWC) systems [3].

Recent work has focused on three of the main challenges associated with textile MPAs used for BCWC: (i) widening their inherent narrow impedance bandwidth (BW) [4], [5], (ii) increasing their front to back ratio (FTBR) to avoid the degradation of their normal operation due to body proximity [1], [2], [6], and (iii) realizing them in a comfortable and aesthetically pleasing manner.

Manuscript received XXXX XX, XXXX; revised Xxxxx XX, XXXX; accepted XXXX XX, XXXX. Date of publication XXXX XX, XXXX; date of current version XXXX XX, XXXX. This work was supported by the Basque Country government by fully granting Ruben Del-Rio-Ruiz a Pre-Doctoral scholarship to perform the Ph.D. degree. (Corresponding author: Ruben DelRio-Ruiz.)

R. Del-Rio-Ruiz, J.-M. Lopez-Garde, and J. Legarda are with University of Deusto - DeustoTech, 48007 Bilbao, Spain (e-mail: ruben.delrio@deusto.es; jmlopez@deusto.es; jlegarda@deusto.es)

O. Caytan, and H. Rogier are with Ghent University - imec, Dept. Information Technology, Technologiepark Zwijnaarde 126, B-9052 Ghent, Belgium (e-mail: olivier.caytan@ugent.be; hendrik.rogier@ugent.be).
The electromagnetically coupled microstrip rectangular patch antenna (EMCMPA) offers a good performance tradeoff in terms of these three challenges [4], [7]-[11]. Nevertheless, the implementation of analytical prediction models to accurately fix the substrate thickness and the dimensions of the radiating patch, the feed line and the ground plane of an EMCMPA is complex due to the electromagnetic coupling between the feed line and the radiating patch.

There are several design models for EMCMPAs, which can be categorized in two groups: simple analytical models and rigorous full-wave models. The latter involves extensive computational processing, which in most cases does not account for fabrication tolerances or other modifications to the realized prototypes compared to the simulated designs [12], [13]. On the other hand, simple analytical models can provide a good and intuitive understanding of the EM performance of the EMCMPA, combined with a reasonably accurate mathematical formulation. These models typically use the transmission line (TL) model to predict the initial dimensions of at least one part of the EMCMPA, but they do not provide all antenna dimensions.

In [8], only the radiating patch of a textile EMCMPA is modeled based on equations from [14], and they do not analyze the deviation between model and measured resonance frequency. However, excellent impedance BW and FTBR values are achieved for the $2.45 \mathrm{GHz}$ industrial, scientific and medical (ISM) band, even though the antenna's radiation efficiency is not simulated nor measured. In [4], a partial analytical model is presented for a dual stub feed line of an EMCMPA in the $10 \mathrm{GHz}$ band, but the deviation in resonance frequency between model and measurements is not specified. Yet, an excellent BW of $8.4 \%$ is achieved, although the radiation efficiency and FTBR values are not provided. In [15], a partial model is presented for a non-textile RT/Duroidbased EMCMPA, which obtains a maximum deviation in resonance frequency of $7.7 \%$. However, the frequency band is not centered at the $2.45 \mathrm{GHz}$ ISM band, and the gain and radiation efficiency values are not available. In [7] and [9], novel textile EMCMPAs are presented. No analytical models are applied in the design phase, but their performance can be considered as a reference for EMCMPAs. [9] shows very good BW and FTBR values, although the overall thickness is high and the BW is not centered at the $2.45 \mathrm{GHz}$ ISM band. Finally, [7] presents a textile EMCMPA for the $2.45 \mathrm{GHz}$ ISM band, with a relatively small thickness. Gain and radiation efficiency 


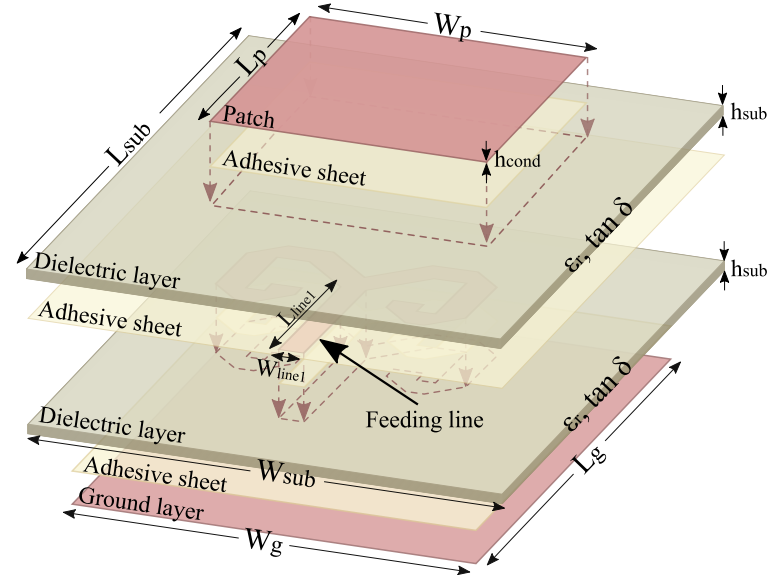

Fig. 1. Perspective view of the electromagnetically coupled microstrip patch textile antenna structure. For clarity, all the antenna layers have been separated and labeled at the side.

values are a good reference for textile EMCMPAs in this band, although no FTBR values are given.

Therefore, this communication presents an analytical design instrument that approximately fixes all dimensions of an EMCMPA, with a given substrate, in the $2.45 \mathrm{GHz}$ ISM band, and with an error lower than that reported in previous publications.

\section{Antenna Design}

\section{A. Antenna Topology and Principle of Operation}

As shown in Fig. 1, the EMCMPA is composed (top to bottom) of a radiating patch $\left(W_{p} \times L_{p} \times h_{\text {cond }}\right)$, a first dielectric layer $\left(W_{\text {sub }} \times L_{\text {sub }} \times h_{\text {sub }}\right)$, a feed line $\left(W_{\text {line } 1} \times\right.$ $\left.L_{\text {line } 1} \times h_{\text {cond }}\right)$, a second dielectric layer $\left(W_{\text {sub }} \times L_{\text {sub }} \times\right.$ $\left.h_{\text {sub }}\right)$, and a ground plane $\left(W_{g} \times L_{g} \times h_{\text {cond }}\right)$.

The EMCMPA is excited by the microstrip feed line, which is electromagnetically coupled through the top substrate to the radiating patch. The feed line is located between the ground plane and the radiating patch, such that any radiation from the feed line is shielded and potentially re-radiated by the patch, leading to a good cross-polarization [4]. This topology places the radiating patch further away from the ground plane (Fig. 2(a)), which leads to enhanced BW and efficiency values, while the feed line is closer to the ground plane to reduce its parasitic radiation.

\section{B. Analytical Model}

The aim of the proposed analytical model is to easily obtain all the dimensions for the preliminary design of an EMCMPA, yielding a resonance frequency as close as possible to the desired value. Moreover, the model also attempts to maximize BW without increasing the overall thickness. The model is suitable to design both rigid and textile based EMCMPA, providing high radiation efficiencies and good antenna performance in close body proximity.

The analytical model combines several TL model equations. First of all, the conventional TL model represents the MPA by two radiating slots (Fig. 2(a)), each described by a parallel equivalent impedance $Z_{r}$ (with resistance $R_{r}$ and capacitance $C_{r}$ ), as shown in Fig. 2(b) [14], and separated by a lowimpedance transmission line ( $T L_{1}$ and $\left.T L_{2}\right)$ of total length

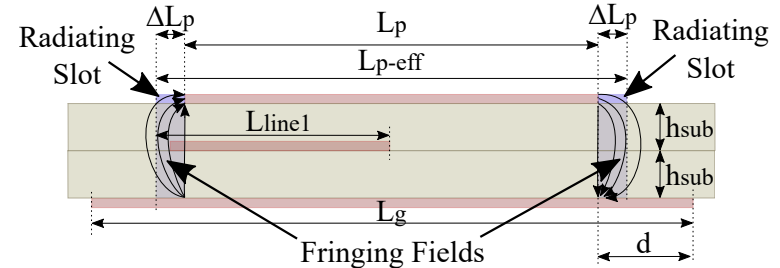

a)

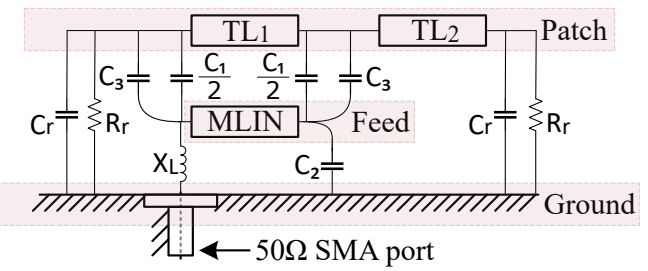

b)

Fig. 2. a) Physical and effective lengths, and b) equivalent circuit model of electromagnetically coupled microstrip patch antennas [16].

$L_{p}$. The dimensions $L_{p}$ and $W_{p}$ of the rectangular microstrip patch are obtained using [14]

$$
\begin{gathered}
W_{p}=\frac{c}{2 f_{r} \sqrt{\left(\varepsilon_{r}+1\right) / 2}} \\
L_{p}=\frac{c}{2 f_{r} \sqrt{\varepsilon_{r e f f}}}-2 \Delta L_{p},
\end{gathered}
$$

where $f_{r}$ is the antenna's desired resonance frequency, $\Delta L_{p}$ is the extension of the patch length at each end caused by the fringing effects of the radiating slots, and $\varepsilon_{r e f f}$ is the effective relative permittivity [14].

Second, the feed line of an EMCMPA can be modeled as a microstrip line (MLIN) [15], which is buried under $T L_{1}$, as shown in Fig. 2(b). $C_{1}$ represents the coupling capacitance between the feed line and the radiating patch, modeled as two capacitors of equal value on either side of MLIN [16]. The feed line exhibits fringing fields, whose effect results in a small increase in its length. This creates extra capacitance, modeled by $C_{2}$, from the open-ended MLIN to the ground, and $C_{3}$, from both sides of MLIN to the radiating patch, which modifies its input impedance, yet the resonance frequency remains constant [16]. The $50 \Omega$ SMA connector is represented in the EMCMPA's equivalent circuit model by an inductive reactance $X_{L}$ connected to MLIN.

As reported in [12], [17], [18], the optimum electromagnetic coupling is obtained when the patch is centered over the end of the feed line and located above it, if the overall thickness $2 \cdot h_{s u b}$ is at a certain critical value [17]. In this communication, the overall thickness is fixed and below the critical value. Therefore, the feed line and patch are over-coupled, which slightly decrements its matching performance [17]. Yet, in these publications, the radiating elements are half-wavelength long dipoles, which leads to quarter-wavelength feed lines, as described in [17]. Therefore, our model suggests a quarterwavelength feed line using

$$
L_{\text {line } 1}=\frac{c}{f_{r \sqrt{\varepsilon_{r e f f}}} \cdot 4}
$$




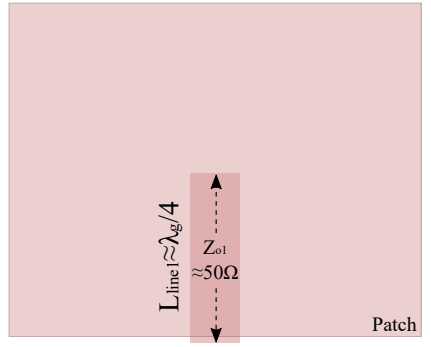

a)

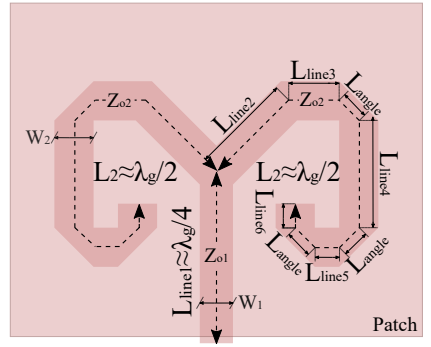

b)
Fig. 3. Proposed feed lines for the electromagnetically coupled microstrip patch antennas. a) Quarter-wavelength feed line. b) Ram-horn shaped feed line [4].

with the open-end at $L_{p} / 2$ and equal substrate thicknesses, as shown in Fig. 2(a). In this communication, the feed line's fringing fields are neglected, since the input impedance's variation is negligible, mainly due to the substrate's small thicknesses and low permittivities. Nevertheless, they should be considered if different dielectric substrates are chosen.

The characteristic impedance of the feed line is set to $Z_{0}=50 \Omega$, as shown in Fig. 3(a). It is approximately calculated using (4) and (5) reported by [19] on page 773, where $Z_{0} \sqrt{\varepsilon_{\text {reff }}} \leq 89.91$.

$$
\begin{gathered}
W_{\text {line } 1}=\frac{2 \cdot h_{\text {sub }}}{\pi} \cdot\left(B-1-\ln (2 B-1)+\frac{\varepsilon_{r}-1}{2 \varepsilon_{r}}\right. \\
\left.\cdot\left(\ln (B-1)+0.39-\frac{0.61}{\varepsilon_{r}}\right)\right) \\
B=\frac{60 \pi^{2}}{Z_{0} \sqrt{\varepsilon_{r}}}
\end{gathered}
$$

Employing a quarter-wavelength feed line allows to cover the desired bandwidth, although in a tight way (2.4 $2.485 \mathrm{GHz}$ ) in the case of the proposed antenna prototypes due to their small substrate thickness. Resonance frequency shifts up to $50 \mathrm{MHz}$ in textile antennas have been reported due to manual Sub-miniature version A (SMA) connectorization and inhomogeneities in the substrate's dielectric properties [20]. Therefore, to better guarantee that the textile antennas cover the entire $2.45 \mathrm{GHz}$ ISM band, a ram-horn shaped feed line is proposed as an alternative design to enlarge the EMCMPA's BW [4]. The ram-horn feed line is a combination of quarterwavelength feed line and two symmetric half-wavelength open-ended stubs connected at $L_{p} / 2$, as shown in Fig. 3(b), that use impedance transformations to achieve greater $\mathrm{BW}$. The initial dimensions of the ram-horn shaped line are obtained from [4], where $L_{\text {Line1 }}$ is a quarter-wavelength long with characteristic impedance $Z_{o 1}$, and $L_{2}$ is a half-wavelength long with characteristic impedance $Z_{o 2}$. The input impedance $Z_{\text {line }}$ of the ram-horn shaped feed line is

$$
Z_{\text {line }} \simeq j Z_{o 1}\left\{\frac{2 \cdot Z_{o 1}}{Z_{o 2}}\right\} \cdot \tan \left(\beta \cdot L_{2}\right)-\cot \left(\beta \cdot L_{\text {line } 1}\right) \text {. }
$$

The ground plane dimensions are modeled using $X_{g}=X_{p}+12 \cdot h_{s u b}$, where $X_{g}$ and $X_{p}$ correspond to either the width $(W)$ or length $(L)$ of the ground and patch, respectively. To ensure that the resonance frequency of the antenna remains almost identical as in an infinite ground plane scenario, the ground plane dimensions must also fulfill the design rule $d \geq \lambda_{0} / 20$ [21], with $d$ being the ground plane extension, as illustrated in Fig. 2(a). In case of conflict the later takes precedence.

The substrate dimensions are obtained by adding $12 \mathrm{~mm}$ to the ground plane, to ease the layer-alignment process. Nevertheless, it has been verified that the antenna performance remains identical when the ground and substrate dimensions are equal.

\section{Antenna Materials}

The proposed analytical model is validated for both rigid and textile materials. The rigid antenna uses FR4 as dielectric layer because its loss tangent is similar to that of textile substrates, and manufacturing with this material is fast and cost-effective by means of a milling-machine [22]. Therefore, a rigid antenna implemented with this material serves as a good validation prior to constructing the textile antennas. The conductive layer is a $35 \mu \mathrm{m}$-thick annealed copper sheet. Both dielectric layers are assembled using plastic nuts and screws. All computer-aided FR4 antenna design models include airgaps and extra FR4 removal gaps to model the removal process of the anneal copper. The extra FR4 removal gaps depend on the prototyping machine's accuracy, which in some cases could be negligible. The textile antenna uses felt as dielectric layer and pure copper polyester taffeta fabric (PCPTF) as conductive layer with a thickness of $80 \mu \mathrm{m}$ and a surface density of $80 \mathrm{~g} / \mathrm{m}^{2}$. Table I summarizes these parameters.

TABLE I

Material Properties of the Two Proposed Antennas

\begin{tabular}{lllllll}
\hline \hline Code & Substrate & $\begin{array}{l}\text { Thickness } \\
(\mathrm{mm})\end{array}$ & $\epsilon_{r}^{\prime}$ & $\begin{array}{l}\text { Loss } \\
\text { T. }\end{array}$ & Conductor & $\begin{array}{l}\text { Sheet Res. } \\
\Omega / s q\end{array}$ \\
\hline$R I G_{x}$ & FR4 & 1.43 & 4.08 & 0.01 & $\begin{array}{l}\text { Annealed } \\
\text { copper }\end{array}$ & 0.0005 \\
\hline$T E X_{x}$ Felt & 1.2 & 1.25 & 0.003 & PCPTF & 0.05 \\
\hline \multicolumn{6}{l}{$\epsilon_{r}^{\prime}$ : optimized relative permittivity at 2.45 GHz. }
\end{tabular}

The preliminary values for the permittivity and loss tangent, shown in Table I, are measured using the Agilent 85070E Dielectric Probe Kit, and the substrate parameters are obtained after a conventional design, simulation and measurement process [23], [24].

The textile antennas are constructed according to the process explained in [20]. A total of four double-sided thermally activated adhesive sheets (approximate thickness of $20 \mu \mathrm{m}$, $\epsilon_{r}=1.74$ and $\left.\tan \delta=0.015\right)$ are required to assemble all the EMCMPA layers, as shown in Fig. 1.

\section{ANTENNA IMPLEMENTATION}

The proposed analytical model is the first step in a three step process to obtain the desired antenna design in a single construction attempt, where the third phase is only necessary if the desired BW is not fully covered. It provides the initial dimensions of all EMCMPA layers for a given resonance frequency, input impedance and dielectric materials. This initial design is optimized in two subsequent phases to center 
the resonance frequency and increase the BW to the desired band. These two optimization steps are performed using the time-domain solver of CST Microwave Studio. Although the proposed design process is intended to avoid manufacturing prototypes until all three steps are completed, in this work the antennas are simulated, manufactured and measured after each phase to see the error evolution between the analytical model and each of the prototypes.

\section{A. Model Implementation}

The proposed analytical model is applied to design an antenna with a resonance frequency of $2.44 \mathrm{GHz}$, an input impedance of $50 \Omega$ and the material parameters described in Table I. The initial dimensions obtained for rigid and textile antennas are shown in Table II, which are designated as $R I G_{1}$ and $T E X_{1}$. The first three letters are denoted by the dielectric layer of the antennas ( $R I G_{1}$ stands for the FR4-based rigid antenna and $T E X_{1}$ stands for the Felt-based textile antenna), and the following number by the design phase $(1-3)$.

TABLE II

Antenna Dimensions Proposed By Analytical Model

\begin{tabular}{lllllllll}
\hline \hline Code & $W_{p}$ & $L_{p}$ & $W_{g}$ & $L_{g}$ & $W_{\text {sub }}$ & $L_{\text {sub }}$ & $W_{\text {line } 1}$ & $L_{\text {line } 1}$ \\
\hline$R I G_{1}$ & 38.57 & 29.49 & 55.73 & 46.65 & 67.73 & 58.65 & 2.89 & 16.07 \\
\hline$T E X_{1}$ & 57.96 & 52.45 & 72.36 & 66.85 & 84.36 & 78.85 & 5.2 & 27.75 \\
\hline \hline
\end{tabular}

Units: $\mathrm{mm}$

The resonance frequency errors between proposed model and simulation, simulation and measurement, and proposed model and measurement are shown in Table III, using (7).

$$
\text { Model vs. Meas. }(\%)=\left|\frac{\left(f r_{\text {model }}-f r_{\text {meas. }}\right)}{f r_{\text {model }}}\right|
$$

In addition, there are two effects derived from the construction process that are considered in the simulation, although their influence on the results is minimal. The first effect is the influence of $h_{\text {air }}$ in rigid antennas, which represents the air layer between the FR4 dielectric layers and the copper conductors due to the tolerance of the milling machine. This air layer $(0.1 \mathrm{~mm})$ causes a decrease of 0.3 to $0.5 \mathrm{~dB}$ in the $\left|S_{11}\right|$ due to the difference in permittivity between FR4 and air. The second effect is due to the adhesive sheet layer $h_{\text {glue }}$ that is used in textile antennas to assemble all layers. This layer causes an amplitude variation of about 1 to $3 \mathrm{~dB}$ and a frequency shift between 2 and $4 \mathrm{MHz}$, due to the higher permittivity of the adhesive and the increase of the total thickness, respectively.

TABLE III

PROPOSED MODEL, SIMULATION, AND MEASUREMENT ERROR RATIOS

\begin{tabular}{lccc}
\hline \hline Code & Model vs. Sim. & Sim. vs. Meas. & Model vs. Meas. \\
\hline$R I G_{1}$ & $4.92 \%$ & $0.43 \%$ & $\mathbf{5 . 3 3 \%}$ \\
\hline$T E X_{1}$ & $2.25 \%$ & $0.63 \%$ & $\mathbf{2 . 8 7 \%}$ \\
\hline$R I G_{2}$ & $0 \%$ & $0.05 \%$ & $\mathbf{0 . 0 5 \%}$ \\
\hline$T E X_{2}$ & $0 \%$ & $0.41 \%$ & $\mathbf{0 . 4 1 \%}$ \\
\hline$R I G_{3}$ & $0.37 \%$ & $0.04 \%$ & $\mathbf{0 . 3 3 \%}$ \\
\hline$T E X_{3}$ & $0.57 \%$ & $0.24 \%$ & $\mathbf{0 . 3 3 \%}$ \\
\hline$T E X_{3 \text { (Onbody) }}$ & $0.45 \%$ & $0.16 \%$ & $\mathbf{0 . 6 2 \%}$ \\
\hline \hline
\end{tabular}

The error between simulation and measurement in both antennas is below $0.63 \%$, which validates the fabrication process reliability for both FR4 and textile antennas [20]. The error ratios between the analytical model's center frequency and the measured resonant frequency in the $R I G_{1}$ and $T E X_{1}$ antennas are $5.33 \%$ and $2.87 \%$, respectively, with a measured BW $\left(\left|S_{11}\right|<-10 d B\right)$ of $3.08 \%$ and $3.32 \%$, respectively. The error ratios achieved with the proposed analytical model are better than those reported in literature [15], [16], providing a good first design approach for EMCMPA.

\section{B. Design Optimization}

A first optimization step is necessary to better adjust the resonance frequency to the desired band. This work proposes to modify only the length of the antenna patch $\left(L_{p}\right)$ in order to tune the antenna to the desired resonance frequency. The new dimensions of $L_{p}$ for the $R I G_{2}$ and $T E X_{2}$ antennas are $27.8 \mathrm{~mm}$ and $51.1 \mathrm{~mm}$, respectively, which cause a resonance frequency shift of $120 \mathrm{MHz}$ and $60 \mathrm{MHz}$, respectively, centering the peak at $2.44 \mathrm{GHz}$. Slightly modifying the length of the radiating patch does not alter the EM performance of the antenna significantly, yielding a similar BW and $\left|S_{11}\right|$ amplitude. The $R I G_{2}$ and $T E X_{2}$ antennas are simulated, manufactured and measured, where a measured BW $\left(\left|S_{11}\right|<-10 d B\right)$ of $3.29 \%$ and $3.49 \%$ is achieved, respectively. The proposed onestep one-variable optimization process provides a maximum resonant frequency error below $0.41 \%$. Nevertheless, as the $2.45 \mathrm{GHz}$ ISM band is tightly covered, a third optimization step is required.

\section{Bandwidth Enhancement}

A ram-horn shaped feed line is proposed to enhance the antenna's BW, as shown in Fig. 3(b). The line widths and lengths of the quarter-wavelength feed line and of the two halfwavelength open-ended stubs are calculated to match $Z_{\text {line }}$ to the $50 \Omega$ input impedance [4]. Afterwards, a computer-aided optimization of the ram-horn shaped feed line is performed using CST Microwave Studio. Applying the design procedure [4] on the $R I G_{2}$ and $T E X_{2}$ prototypes, should yield a BW of 1.4 and 1.6 times the original $3.29 \%$ and $3.49 \%$ BW, respectively. Yet, slightly lower measured BW increments of 1.2 and 1.5 are obtained in $R I G_{3}$ and $T E X_{3}$, respectively, which are in line with the measured increments obtained in [4]. The resonant frequency of $R I G_{3}$ and $T E X_{3}$ antennas is centered at $2.44 \mathrm{GHz}$ and the BW fully covers the $2.45 \mathrm{GHz}$ ISM band, as shown in Fig. 4. The optimized ram-horn shaped feed line dimensions of the antennas are shown in Table IV.

TABLE IV

RAM-HORN SHAPED FEED LiNE DimENSIONS

\begin{tabular}{llllllllll}
\hline \hline Code & $W_{1}$ & $W_{2}$ & $L_{\text {line } 1}$ & $L_{\text {line } 2}$ & $L_{\text {line } 3}$ & $L_{\text {line4 }}$ & $L_{\text {line }}$ & $L_{\text {line6 }}$ & $L_{\text {angle }}$ \\
\hline$R I G_{3}$ & 3.06 & 4.1 & 38.57 & 29.49 & 55.73 & 46.65 & 67.73 & 58.65 & 5.79 \\
\hline$T E X_{3}$ & 3.68 & 7.43 & 57.96 & 52.45 & 72.36 & 66.85 & 84.36 & 78.85 & 10.4 \\
\hline \hline
\end{tabular}

Units: $\mathrm{mm}$

\section{FinAl EXPERIMENTAL VALIDATION}

The electromagnetically coupled ram-horn shaped fed microstrip patch antennas are fabricated and tested under free 


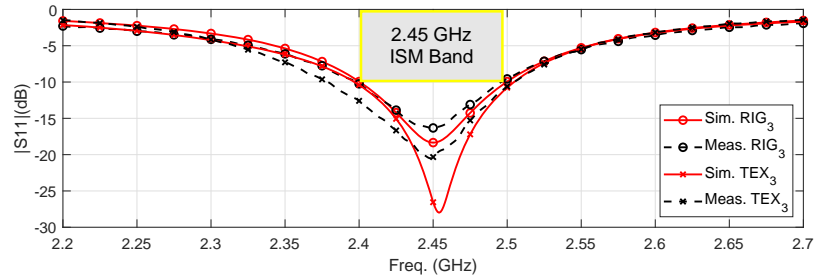

Fig. 4. Simulated and measured reflection coefficient results in free space for $R I G_{3}$ and $T E X_{3}$ electromagnetically coupled microstrip patch antennas, using FR4 and felt dielectric layers.

space and on-body conditions to validate their EM performance. The antenna measurements are performed with an Agilent N5242A PNA-X Microwave Network Analyzer, with the antennas placed in an anechoic chamber.

\section{A. Free Space Performance}

The simulated and measured reflection coefficients of the rigid and textile antennas are shown in Fig. 4. Excellent agreement is observed between simulated and measured values. Both $R I G_{3}$ and $T E X_{3}$ antennas are centered at $2.44 \mathrm{GHz}$ and cover the entire $2.45 \mathrm{GHz}$ ISM band $(2.4-2.4835 \mathrm{GHz})$, with a measured BW $\left(\left|S_{11}\right|<-10 d B\right)$ of $4 \%$ and $5.12 \%$, respectively.

Cross sections of the radiation pattern of the antennas in the E- and $\mathrm{H}$-planes are measured in the anechoic chamber at $2.45 \mathrm{GHz}$, as shown in Fig. 5. Again, there is an excellent agreement between the measured and simulated results. The maximum measured co-polarization gains for the $R I G_{3}$ and $T E X_{3}$ antennas are $4.11 \mathrm{dBi}$ and $7.33 \mathrm{dBi}$, respectively. The cross-polarization is very small, both in simulations and measurements. Their deviations are due to effects by the mounting fixtures of the measurement system and to positioning and measurement errors.

The simulated and measured maximum antenna gain and the total antenna efficiency between $2.4 \mathrm{GHz}$ and $2.5 \mathrm{GHz}$ are plotted in Fig. 6. The maximum measured antenna gains for the $R I G_{3}$ and $T E X_{3}$ antennas equal $4.28 \mathrm{dBi}$ and $7.33 \mathrm{dBi}$, respectively. The maximum measured antenna efficiencies for the $R I G_{3}$ and $T E X_{3}$ antennas equal $63.4 \%$ and $71.8 \%$, respectively. There is a good agreement between the measured and simulated $R I G_{3}$ antenna results, while the textile antenna has a difference of less than $10 \%$ due to slight variations in the felt dielectric properties.

\section{B. On-Body Performance}

The $T E X_{3}$ textile antenna is simulated and measured on the torso of an average adult person. The simulation uses a three layer (skin-fat-muscle) body phantom. The dielectric properties of the phantom layers are obtained from the Hugo voxel model from the CST material library. The two main phantom thickness setups [2], [25] are simulated to obtain a better accuracy, with overall thicknesses of 22 and $70 \mathrm{~mm}$, respectively. The measurement is performed with a person of size $1.75 \mathrm{~m}$ and weight $85 \mathrm{~kg}$ inside the anechoic chamber. In both cases, the antenna is placed $2 \mathrm{~mm}$ away from the simulated phantom and measured body. The simulated and measured reflection coefficients in on-body and free space scenarios are compared in Fig. 7. The reflection coefficient
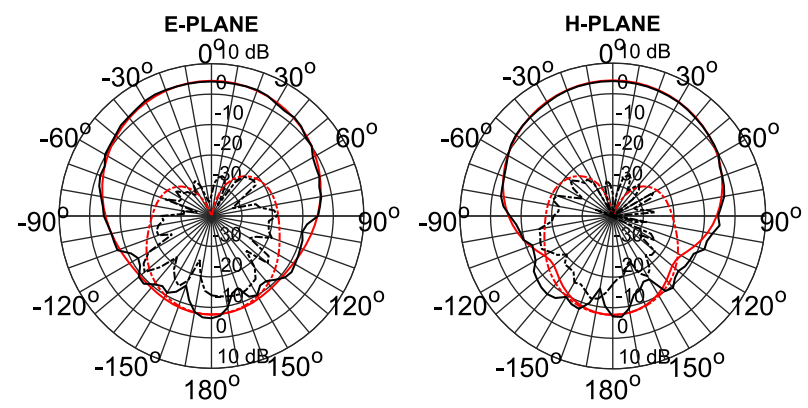

a)
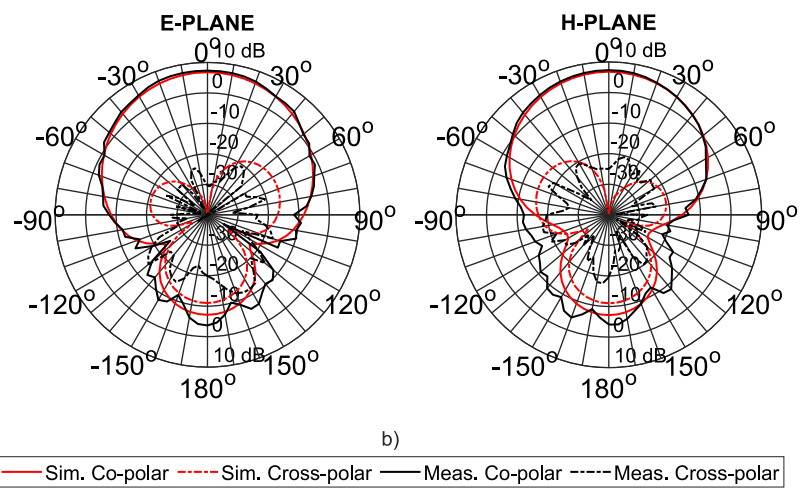

Fig. 5. Simulated and measured co- and cross-polarization patterns in free space for a) $R I G_{3}$ and b) $T E X_{3}$ electromagnetically coupled microstrip patch antennas, using FR4 and felt dielectric layers, respectively.

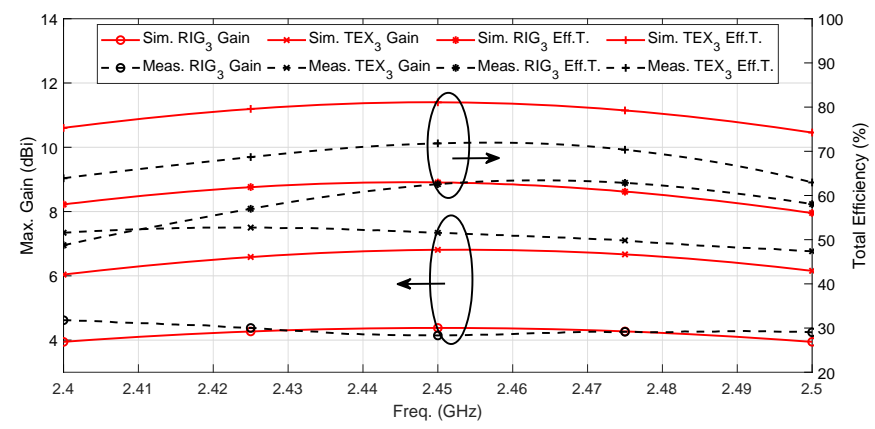

Fig. 6. Simulated and measured maximum gain and total antenna efficiency results in free space for $R I G_{3}$ and $T E X_{3}$ electromagnetically coupled microstrip patch antennas, using FR4 and felt dielectric layers.

remains below $-10 \mathrm{~dB}$ in the entire $2.45 \mathrm{GHz}$ ISM band and the simulation and measurement differences between on-body and free-space scenarios are negligible.

The 3D measurement of the antenna performance on the torso of a person inside the anechoic chamber is not possible. Therefore, the antenna is reoriented to measure the far-field radiation patterns in the E- and H-planes at $2.45 \mathrm{GHz}$. Fig. 8 shows the co- and cross-polarization results, where the maximum gain is $5.5 \mathrm{dBi}$. The measured cross-polarization in the E- and H-plane remains below $-13.2 \mathrm{dBi}$. The patterns in the E- and $\mathrm{H}$-planes at $2.45 \mathrm{GHz}$ agree well both in shape and absolute values when placed on the body, which means that the proposed design method provides also a good performance for BCWC applications.

Finally, the specific absorption rate (SAR) values are simulated using the proposed phantom properties and thicknesses [2], [25], obtaining maximum SAR values at $2.45 \mathrm{GHz}$ 


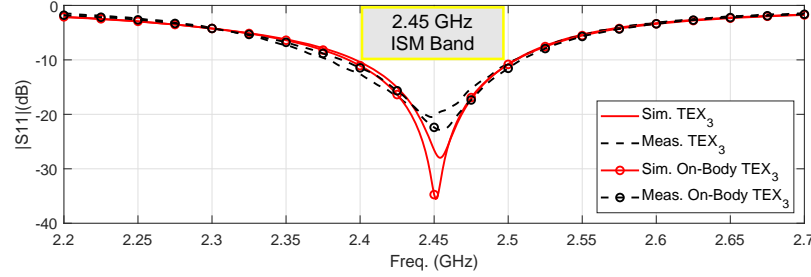

Fig. 7. Simulated and measured on-body reflection coefficient results for the $T E X_{3}$ electromagnetically coupled microstrip patch antennas, using felt dielectric layers.
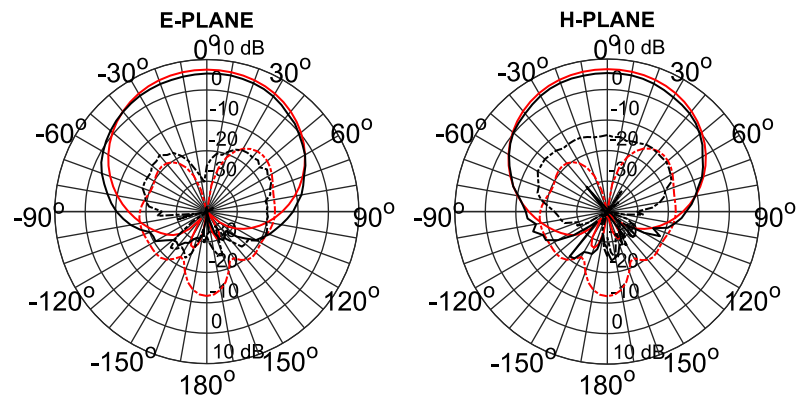

Sim. Co-polar -----Sim. Cross-polar — Meas. Co-polar -----Meas. Cross-polar

Fig. 8. Simulated and measured on-body co- and cross-polarization patterns for the $T E X_{3}$ electromagnetically coupled microstrip patch antenna, using felt dielectric layers.

of $0.199 \mathrm{~W} / \mathrm{kg}$ and $0.323 \mathrm{~W} / \mathrm{kg}$, respectively, which are well below the limit of $1.6 \mathrm{~W} / \mathrm{kg}$, according to standard IEEE C95.3, with an input power of $0.5 \mathrm{~W}$ (rms), and averaged over $1 \mathrm{~g}$ of biological tissue. These results are coherent with the values shown in Fig. 8, which indicate that most of the radiation is directed away from the body, owing to the presence of a ground plane and a shielded feed line.

\section{CONClusion}

The analytical model proposed in this communication quickly and reliably yields the initial dimensions of an EMCMPA. Two subsequent simulation phases which modify a single design variable in each phase are presented, achieving a centered resonance frequency and BW enhancement, respectively. This design instrument enables the realization, in a single attempt, of an EMCMPA antenna for the $2.45 \mathrm{GHz}$ ISM band on both rigid and textile materials. The textile antennas designed with this procedure have a high flexibility owing to their small thickness, and an excellent radiation performance in BCWC applications, with antenna gains and efficiencies of $7.33 \mathrm{dBi}$ and $71.8 \%$, respectively, and SAR values lower than $0.4 \mathrm{~W} / \mathrm{kg}$.

\section{REFERENCES}

[1] H. Lee, J. Tak, and J. Choi, "Wearable antenna integrated into military berets for indoor/outdoor positioning system," IEEE Antennas and Wireless Propagation Letters, vol. 16, pp. 1919-1922, 2017.

[2] S. Agneessens and H. Rogier, "Compact half diamond dual-band textile HMSIW on-body antenna," IEEE Transactions on Antennas and Propagation, vol. 62, no. 5, pp. 2374-2381, May 2014.

[3] K. F. Lee, K. M. Luk, and H. W. Lai, Microstrip Patch Antennas, 2nd ed. River Edge, NJ, USA: World Scientific Publishing Co., Inc., 2017.

[4] S. M. Duffy, "An enhanced bandwidth design technique for electromagnetically coupled microstrip antennas," IEEE Transactions on Antennas and Propagation, vol. 48, no. 2, pp. 161-164, Feb 2000.
[5] D. Guan, Y. Zhang, Z. Qian, Y. Li, W. Cao, and F. Yuan, "Compact microstrip patch array antenna with parasitically coupled feed," IEEE Transactions on Antennas and Propagation, vol. 64, no. 6, pp. 25312534, June 2016.

[6] M. Virili, H. Rogier, F. Alimenti, P. Mezzanotte, and L. Roselli, "Wearable textile antenna magnetically coupled to flexible active electronic circuits," IEEE Antennas and Wireless Propagation Letters, vol. 13, pp. 209-212, 2014.

[7] S. Adami, P. Proynov, G. S. Hilton, G. Yang, C. Zhang, D. Zhu, Y. Li, S. P. Beeby, I. J. Craddock, and B. H. Stark, "A flexible $2.45-\mathrm{GHz}$ power harvesting wristband with net system output from $-24.3 \mathrm{dBm}$ of RF power," IEEE Transactions on Microwave Theory and Techniques, vol. 66, no. 1, pp. 380-395, Jan 2018.

[8] M. Grilo, M. Hiroaki Seko, and F. Salete Correra, "Wearable textile patch antenna fed by proximity coupling with increased bandwidth," Microwave and Optical Technology Letters, vol. 58, no. 8, pp. 19061912, 2016.

[9] B.-L. Ooi, "A double- $\pi$ stub proximity feed U-slot patch antenna," IEEE Transactions on Antennas and Propagation, vol. 52, no. 9, pp. 24912496, Sep 2004.

[10] R. Del-Rio-Ruiz, J. Lopez-Garde, and J. L. Macon, "Design and performance analysis of a purely textile proximity fed microstrip patch antenna for on-body wireless communications," in 2018 IEEE International Symposium on Antennas and Propagation USNC/URSI National Radio Science Meeting, July 2018, pp. 1293-1294.

[11] R. Del-Rio-Ruiz, J.-M. Lopez-Garde, and J. Legarda, "Planar textile off-body communication antennas: A survey," Electronics, vol. 8, no. 6, 2019.

[12] P. Katehi and N. Alexopoulos, "On the modeling of electromagnetically coupled microstrip antennas-the printed strip dipole," IEEE Transactions on Antennas and Propagation, vol. 32, no. 11, pp. 1179-1186, November 1984.

[13] P. Lepeltier, J. M. Floc'h, and J. Citerne, "Complete and rigorous analysis of electromagnetically coupled transverse microstrip dipole," Electronics Letters, vol. 23, no. 16, pp. 822-824, July 1987.

[14] C. A. Balanis, Antenna theory: analysis and design. Wiley-Interscience, 2005.

[15] B. Belentepe, "Modeling and design of electromagnetically coupled microstrip-patch antennas and antenna arrays," IEEE Antennas and Propagation Magazine, vol. 37, no. 1, pp. 31-39, Feb 1995.

[16] S. Vajha and S. N. Prasad, "Design and modeling of proximity coupled patch antenna," in 2000 IEEE-APS Conference on Antennas and Propagation for Wireless Communications, Nov 2000, pp. 43-46.

[17] H. Oltman and D. Huebner, "Electromagnetically coupled microstrip dipoles," IEEE Transactions on Antennas and Propagation, vol. 29, no. 1, pp. 151-157, January 1981.

[18] D. M. Pozar and B. Kaufman, "Increasing the bandwidth of a microstrip antenna by proximity coupling," Electronics Letters, vol. 23, no. 8, pp. 368-369, April 1987.

[19] R. Garg, P. Bhartia, I. Bahl, and A. Ittipiboon, Microstrip antenna design handbook, ser. Artech House Antennas and Propagation Library. Boston: Artech House, 2001.

[20] R. Del-Rio-Ruiz, J. Lopez-Garde, J. Legarda, S. Lemey, O. Caytan, and H. Rogier, "Reliable lab-scale construction process for electromagnetically coupled textile microstrip patch antennas for the $2.45 \mathrm{GHz}$ ISM band," IEEE Antennas and Wireless Propagation Letters, vol. 19, no. 1, pp. 153-157, Jan 2020.

[21] E. Lier and K. Jakobsen, "Rectangular microstrip patch antennas with infinite and finite ground plane dimensions," IEEE Transactions on Antennas and Propagation, vol. 31, no. 6, pp. 978-984, November 1983.

[22] Laser and Electronics, "Protomat S62." [Online]. Available: http: //www.lpkfusa.com/datasheets/prototyping/s62.pdf

[23] F. Declercq, I. Couckuyt, H. Rogier, and T. Dhaene, "Complex permittivity characterization of textile materials by means of surrogate modeling," in 2010 IEEE Antennas and Propagation Society International Symposium, July 2010, pp. 1-4.

[24] S. Sankaralingam and B. Gupta, "Determination of dielectric constant of fabric materials and their use as substrates for design and development of antennas for wearable applications," IEEE Transactions on Instrumentation and Measurement, vol. 59, no. 12, pp. 3122-3130, Dec 2010.

[25] S. Yan, P. J. Soh, and G. A. E. Vandenbosch, "Low-profile dualband textile antenna with artificial magnetic conductor plane," IEEE Transactions on Antennas and Propagation, vol. 62, no. 12, pp. 6487 6490, 2014 